Physica B 163 (1990) 689-691

North-Holland

\title{
POSSIBLE MECHANISMS FOR NONLINEAR DE HAAS-VAN ALPHEN OSCILLATIONS IN HEAVY FERMION COMPOUNDS
}

\author{
J.W. RASUL ${ }^{a}$ and P. SCHLOTTMANN ${ }^{\mathrm{b}}$ \\ ${ }^{2}$ Department of Physics, University of Michigan, Ann Arbor, MI 48109, USA \\ ${ }^{\mathrm{b}}$ Department of Physics, Temple University, Philadelphia, PA 19122, USA
}

\begin{abstract}
Three possible many-body mechanisms giving rise to de Haas-van Alphen oscillations with frequencies that correspond to linear combinations of ordinary closed orbits of the Fermi surface are discussed in the context of heavy-fermion systems. The mechanisms are: (i) magnetic breakdown across the Kondo hybridization gap (magnetically induced tunneling), (ii) the strong nonlinear dependence of the heavy-fermion magnetization of the magnetic field may give rise to frequency mixing (Shoenberg effect), and (iii) the incipient antiferromagnetic order in many heavy-fermion compounds at low $T$ provides a nonlinear coupling mechanism via modulation of the antiferromagnetic order parameter. The latter should be very effective in weak antiferromagnets.
\end{abstract}

\section{Introduction}

The observation of the de Haas-van Alphen effect in several heavy fermion compounds $\left(\mathrm{CeCu}_{6}[1], \mathrm{UPt}_{3}\right.$ [2], $\mathrm{CeB}_{6}$ [3]) has dramatically demonstrated the existence of a Fermi surface and of quasiparticles with a strongly enhanced mass. The dHvA oscillations have been successfully interpreted in terms of Fermi surfaces arising from band-structure calculations. Since many-body correlations are only partially included in band-structure calculations, the resulting effective masses are not heavy enough. The Fermi surface, on the other hand, is essentially determined by the symmetry of the system and Luttinger's theorem [4].

There have been several attempts to incorporate the heavy masses into the band picture. A phenomenological approach, devised by Razafimandimby et al. [5], builds in the correct effective mass into an appropriate resonant level phase shift of a KKR band-structure calculation. Microscopic derivations of the quasiparticle behavior within the framework of the Anderson lattice have been incorporated into the exponential dependence of the dHvA amplitude on the effective mass by various authors [6-9]. Rasul [8] has shown that the effective mass in the $\mathrm{dHvA}$ amplitude is the same as the one entering the specific heat coefficient $\gamma$. It is also concluded that the Engelsberg-Simpson expression [10] for the dHvA amplitude remains valid despite of the high correlations among the electrons.

In this paper we present a qualitative discussion of possible many-body mechanisms which may produce nonlinear dHvA oscillations in heavy-fermion systems, i.e., oscillations with frequencies that are linear combinations of frequencies of ordinary oscillations (corresponding to closed orbits of the Fermi surface). The new oscillations then do not correspond to an extremal closed cross section of the Fermi surface. We discuss the following three mechanisms in the context of heavy fermion compounds to show that they may give rise to observable effects: (i) magnetic breakdown [11], (ii) the Shoenberg effect [12], and (iii) modulation of the antiferromagnetic order parameter [13].

Since the quasiparticle picture appears to be valid, we consider quasiparticles with a very large mass enhancement. Herewith it is implicitly assumed that only low energy excitations in the Fermi liquid are relevant to the dHvA oscillations. To simplify our arguments we assume a nearly free electron Fermi surface. The orbits of the heavy electrons in a small magnetic field then consist of piecewise circular orbits and Bragg reflections at the Brillouin zone boundaries.

\section{Magnetic breakdown}

Trajectories of electrons in $k$ space are defined by the intersections of planes perpendicular to the magnetic field $\boldsymbol{H}$ and surfaces of constant energy, in particular with the Fermi surface. Nearly free electrons move along circular orbits until they are Bragg reflected at the Brillouin zone boundary. Within the reduced zone scheme the Bragg reflections confine an orbit to one Brillouin zone and boundaries cannot be 
crossed. As a consequence of the Bragg reflections a gap opens at the zone boundaries. A sufficiently strong magnetic field can force the electrons to continue their circular orbit across the zone boundary, i.e., to tunnel from one Fermi surface orbit to another. In other words, a strong magnetic field changes the connectivity of the orbits. This effect is known as magnetic breakdown [14]. The tunneling probability between two orbits depends exponentially on the inverse of the field, $P \approx \exp \left(-H_{0} / H\right)$, where $H_{0}$ is approximately $4 \pi E_{\mathrm{G}}^{2} c / v_{\mathrm{F}}^{2} \hbar|e|$ and $E_{\mathrm{G}}$ is the gap. The gap is given by the corresponding Fourier-component of the crystal potential (independent of the effective mass) and $v_{\mathrm{F}}$ is inversely proportional to $\mathrm{m}^{*}$. We expect then that $H_{0}$ is very large for heavy fermions and the tunneling process to be ineffective if the electrons have large masses close to the zone boundary.

There is the possibility of another type of magnetic breakdown in heavy fermion compounds, which is not related to Bragg reflections at the Brillouin zone boundaries. Within the molecular field framework of the Anderson lattice [15], the hybridization matrix element $V$ of the conduction and quasilocalized states is strongly renormalized, $\tilde{V}=V\left(1-n_{\mathrm{f}}\right)^{1 / 2}$, and is very small in the Kondo limit, $n_{\mathrm{f}} \rightarrow 1$. In the absence of hybridization there are two independent Fermi surfaces, one for the conduction electrons, the other corresponding to the highly correlated states (assumed here to have a small dispersion). The hybridization introduces a gap and modifies the topology of the Fermi surface at the points (we consider planes perpendicular to the magnetic field) where the two Fermi surfaces intersect. In weak field the electrons follow the orbits of the hybridized Fermi surface. In a very strong magnetic field, on the other hand, the electrons move in orbits of the Fermi surface without hybridization. The competition of two energies, the hybridization gap $\tilde{\boldsymbol{V}}$ (or the Kondo energy) and the cyclotron frequency (with heavy mass $m^{*}$ ), determine the tunneling process at intermediate fields (i.e., still large fields in the lab).

\section{The Shoenberg effect}

In the classical theory of the dHvA effect the applied field $\boldsymbol{H}$ gives rise to oscillatory contributions to the magnetization where each frequency appears together with its higher harmonics. In the presence of electron interactions (i.e., beyond the independent electron approximation) the electrons experience the total magnetic induction, $\boldsymbol{B}=\boldsymbol{H}+4 \pi \boldsymbol{M}$, rather than the applied field $\boldsymbol{H}$. As has been shown by Shoenberg [12] and Pippard [16] this gives rise to a nonlinear self-consistent equation for the magnetization. The magnetization has oscillatory and nonoscillatory components. Let us assume first that there is only one relevant cross section of the Fermi surface and that the system is not close to a ferromagnetic instability. The self-consistent solution then modifies the content of the higher harmonics, i.e., their amplitudes are changed.

If two or more closed orbits are involved the selfconsistent solution will lead to a mixing of frequencies, i.e., to frequencies corresponding to cross sections $A_{1} \pm A_{2}$ and in principle to any linear combination of them. If $A_{1}$ and $A_{2}$ are of the same order $\left|A_{1}-A_{2}\right|$ is small compared to $A_{1}$ and $A_{2}$ (and their harmonics), so that it is in a new region of the spectrum where it could easily be detected. The amplitude of the mixed frequency $\left|A_{1}-A_{2}\right|$ is expected to be small and its temperature dependence associated with the sum of the effective masses corresponding to $A_{1}$ and $A_{2}$, $m_{1}+m_{2}$. This magnetic interaction effect has been observed in various metals, e.g., in $\mathrm{Ag} \mathrm{[17]} \mathrm{and} \mathrm{In}$ [18].

Heavy fermion systems typically have a very large susceptibility (note that the Wilson ratio $\chi / \gamma$ is approximately the one of free electrons). This large magnetic response is mainly due to the spin paramagnetism of the f-electrons, rather than caused by the quantization of the electron orbits. The oscillatory component of the magnetization represents then a small fraction of the large susceptibility. However, we expect the frequency mixing content due to magnetic interactions to be large enough to be observable despite of the heavy masses involved.

\section{Modulation of the antiferromagnetic order parameter}

Incipient antiferromagnetic order with a very small ordered magnetic moment has been found in some heavy fermion compounds, e.g., $\mathrm{UPt}_{3}[19], \mathrm{CeCu}_{6}[20]$ and $\mathrm{CeAl}_{3}$ [21]. The presence of antiferromagnetic order, or an order parameter in general, gives rise to another mechanism [13] for the nonlinear coupling of $\mathrm{dHvA}$ frequencies. Long-range order in general modifies the properties of the Fermi surface. This is selfconsistently determined by the many-body collective properties of the metal via the energy-gap or order parameter $\Delta$ which satisfies a nonlinear integral equa- 
tion (the gap equation or Ginzburg-Landau functional). The presence of a magnetic field (i.e., the quantization of the orbits) modifies the kernel of the integral equation. Hence, the order parameter $\Delta$ has an oscillatory contribution which affects the one-electron energy spectrum and as a consequence the $\mathrm{dHvA}$ oscillation spectrum.

Neglecting the Landau diamagnetism, the free energy can be written as $F(\Delta, H)=F_{c}(\Delta, H)+$ $F_{\text {osc }}(\Delta, H)$, where $F_{\text {c }}$ is the Ginzburg-Landau energy and $F_{\text {osc }}$ is responsible for the $\mathrm{dHvA}$ oscillations. The equilibrium value of $\Delta(H)$ is obtained by minimizing the free energy with respect to $\Delta, \Delta(H)=\Delta_{c}(H)+$ $\delta \Delta(H)$, where $\Delta_{\mathrm{c}}$ is the nonoscillatory contribution and

$\delta \Delta(H)=-\left(\mathrm{d} F_{\mathrm{osc}} / \mathrm{d} \Delta\right)_{\Delta_{\mathrm{c}}} /\left(\mathrm{d}^{2} F_{\mathrm{c}} / \mathrm{d} \Delta^{2}\right)_{\Delta_{\mathrm{c}}}$.

Hence, to second order the free energy is

$$
\begin{aligned}
F= & F_{\mathrm{c}}\left(\Delta_{\mathrm{c}}, H\right)+F_{\mathrm{osc}}\left(\Delta_{\mathrm{c}}, H\right) \\
& -\frac{1}{2}\left[\left(\mathrm{~d} F_{\mathrm{osc}} / \mathrm{d} \Delta\right)_{\Delta_{\mathrm{c}}}\right]^{2} /\left(\mathrm{d}^{2} F_{\mathrm{c}} / \mathrm{d} \Delta^{2}\right)_{\Delta_{\mathrm{c}}} .
\end{aligned}
$$

The last term gives rise to oscillatory terms which vary periodically with inverse magnetic field with frequencies that are characteristic of areas $\left(n_{i} A_{i} \pm n_{j} A_{j}\right)$. In all cases the areas are for $\Delta=\Delta_{\mathrm{c}}$, and $n_{i}$ and $n_{j}$ are integers. Hence, antiferromagnetic order can modify the harmonic content of the oscillations, but the main effect is the appearance of new frequencies. The amplitudes of the nonlinear effect are inversely proportional to $a=\mathrm{d}^{2} F_{\mathrm{c}} / \mathrm{d} \Delta^{2}$. Since $a$ is a measure of the stability of the order $\approx\left(T-T_{\mathrm{c}}\right)$ (close to $\left.T\right)$, and vanishes as $T \rightarrow T_{c}$, the mixing effect should be more easily observable close to the transition.

\section{Summary}

In summary, we have pointed out three possible sources of nonlinearity in $\mathrm{dHvA}$ measurements of heavy-fermion systems, namely magnetic breakdown, the Shoenberg effect, and feedback effects related to the antiferromagnetic order parameter. The latter two processes should produce frequencies corresponding to the difference between Fermi surface cross sectional areas.
We acknowledge the support by the US Department of Energy under grant DE-FG02-87ER45333.

\section{References}

[1] P.H.P. Reinders, M. Springford, P.T. Coleridge, R. Boulet and D. Ravot, Phys. Rev. Lett. 57 (1986). J. Magn. Magn. Mat. 63 \& 64 (1987) 297.

[2] L. Taillefer, R. Newbury, G.G. Lonzarich, Z. Fisk and J.L. Smith, J. Magn. Magn. Mat. 63 \& 64 (1987) 372.

[3] W. Joss, J.M. van Ruitenbeek, G.W. Crabtree, J.L. Tholence, A.J.P. van Deursen and Z. Fisk, Phys. Rev. Lett. 59 (1987) 1609.

[4] R.M. Martin, Phys. Rev. Lett. 48 (1982) 362.

[5] H. Razafimandimby, P. Fulde and J. Keller, Z. Phys. B 54 (1984) 111.

[6] P.C.E. Stamp, Europhys. Lett. 4 (1987) 453.

[7] P. Thalmeier, J. Phys. C 20 (1987) L899.

[8] J.W. Rasul, Phys. Rev. B 39 (1989) 663.

[9] A. Wasserman, M. Springford and A.C. Hewson, J. Phys. Cond. Mat. 1 (1989) 2669.

[10] S. Engelsberg and G. Simpson, Phys. Rev. B 2 (1970) 657.

[11] L.M. Falicov, in: Electrons in Crystalline Solids: Trieste Lectures, 1972 (UNIPUB, New York, 1973) p. 207.

[12] D. Shoenberg, Philos. Trans. Roy. Soc. London, Ser. A 255 (1963) 85.

[13] P. Schlottmann and L.M. Falicov, Phys. Rev. 38 (1977) 855.

[14] M.H. Cohen and L.M. Falicov, Phys. Rev. Lett. 7 (1961) 231.

[15] D.M. Newns and N. Read, Adv. Phys. 36 (1987) 799.

[16] A.B. Pippard, Proc. Roy. Soc. London, Ser. A 272 (1963) 192.

[17] A.S. Joseph and A.C. Thorsen, Phys. Rev. 138 (1965) A1159.

[18] J.H.P. Van Weeren and J.R. Anderson, J. Phys. F 3 (1973) 2109

[19] J.K. Kjems and C. Broholm, J. Magn. Magn. Mat. 76 \& 77 (1988) 371.

G. Aeppli, E. Bucher, C. Broholm, J.K. Kjems, J. Baumann and J. Hufnagl, Phys. Rev. Lett. 60 (1988) 615.

[20] J. Rossat-Mignod, L.P. Regnault, J.L. Jacoud, C. Vettier, P. Lejay, J. Flouquet, E. Walker, D. Jaccard and A. Amato, J. Magn. Magn. Mat. 76 \& 77 (1988) 376.

[21] S. Barth, H.R. Ott, F.N. Gygax, B. Hitti, E. Lippelt and A. Schenck, J. Magn. Magn. Mat. 76 \& 77 (1988) 455. 